\title{
Cavete zu meinhausarzt.ch/Printus Verlag AG - zum dritten
}

Hanspeter Kuhn, Fürsprecher, stv. Generalsekretär

Korrespondenz: FMH-Rechtsdienst Elfenstrasse 18 CH-3000 Bern 15 Tel. 0313591111 Fax 0313591112

lex@fmh.ch
Der Printus-Verlag mit seinem sogenannten «Ärzteverzeichnis 2006» lässt noch nicht locker. Verschiedene Ärztinnen und Ärzte haben in den letzten Tagen eine Zahlungsaufforderung der Inkassofirma DEBI CONTROL GmbH erhalten. Hingegen haben wir noch nicht gehört, dass der Verlag gegen einen Arzt eine Betreibung eingeleitet hätte.

Zusammenfassung unserer Empfehlung für Empfänger der Zahlungsaufforderung der DEBI CONTROL GmbH (gemäss Stand bei Redaktionsschluss):

- Schreiben Sie dem Verlag erneut - mit Kopie an die Inkassofirma (die gemäss Handelsregister denselben Hauptpersonen gehört ...) mit eingeschriebenem Brief, beispielsweise mit folgendem Text: "Ich halte an meinem Standpunkt fest: Es liegt Grundlagenirrtum und Täuschung vor, der Vertrag ist nichtig bzw. bin- det mich nicht. Gleichzeitig kündige ich vorsorglich jede Weiterführung oder Erneuerung meines Eintrags in Ihrem «Ärzteverzeichnis`.»

- Wenn Sie einen Zahlungsbefehl erhalten, so erheben Sie sofort Rechtsvorschlag. Rechtsvorschlag können Sie unmittelbar bei Erhalt eines Zahlungsbefehls gegenüber dem Betreibungsbeamten erheben und sonst innert 10 Tagen beim Betreibungsamt.

- Blick in die Zukunft: Falls der Verlag versuchen sollte, ein Rechtsöffnungsgesuch einzugeben: Konsultieren Sie diesfalls erneut unsere Website.

Sie finden unsere aktuellen Empfehlungen jeweils auf der Website der FMH (www.fmh.ch; deutsch; der Link zu Printus ist direkt auf der Homepage). 